\title{
SODIC PINATE AS A TEST FOR LIME.
}

By J. A. SEWALL.

Pinic acid or sodic pinate precipitate salts of calcium, magnesium and iron.

I have recently made some experiments with the sodic pinate, with reference to its delicacy as a test for detecting the presence of the salts of the first mentioned metal (calcium), with the following results, using a solution of calcic sulphate:

$\frac{1}{2500}$ part of calcic sulphate in one part of water, yields a very copious amorphous precipitate, which readily subsides.

$\frac{1}{5000}$ part yields an abundant bulky precipitate.

$\frac{1}{10000}$ part, an abundant precipitate.

$\frac{1}{25000}$ part, an immediate cloudiness, and in a few minutes a good precipitate.

$\frac{1}{50000}$ part, quite the same result as is given by the $\frac{1}{2000}$ solution.

$12 \frac{1}{5} 00$ part, a very satisfactory deposit after a little time. deposit.

$2 \frac{1}{0000}$ part, a distinct turbidity, and after a few hours a satisfactory

I observe that on adding a few drops of the reagent to distilled water, the solution becomes, after several days, slightly opalescent.

The reagent was prepared by dissolving one part of the sodic pinate in fifteen parts of distilled water and filtering the solution.

The quantity of the solution of the calcic sulphate operated on in each trial was one fluid ounce. The quantity of the sodic pinate solution used in each trial varied from two to ten drops, the larger quantity being used in the stronger solution of the calcic salt. senius).

Ammonic oxalate fails to precipitate lime in a $\frac{1}{650} \overline{0}$ solution, (Fre-

It will be seen from the above that the sodic pinate is a much more delicate test for calcic salts than the ammonic oxalate. The deportment of other calcic salts is quite the same as that of the sulphate. 